\title{
Adjunctive ketamine for sedation in critically ill mechanically ventilated patients: an active-controlled, pilot, feasibility clinical trial
}

\author{
Marwa Amer ${ }^{1,2^{*}}$ (D) Khalid Maghrabi ${ }^{2,3}$, Mohammed Bawazeer $^{3}$, Kamel Alshaikh ${ }^{3}$, Mohammad Shaban ${ }^{3}$, \\ Muhammad Rizwan ${ }^{3}$, Rashid Amin ${ }^{1}$, Edward De Vol ${ }^{4}$, Mawadah Baali $^{4}$, Malak Altewerki ${ }^{5}$, Mehreen Bano $^{6}$, \\ Fawziah Alkhaldi ${ }^{6}$, Sanaa Alenazi ${ }^{6}$ and Mohammed Hijazi ${ }^{3}$
}

\begin{abstract}
Objective: Ketamine has been shown to decrease sedative requirements in intensive care unit (ICU). Randomized trials are limited on patient-centered outcomes. We designed this pilot trial to evaluate the feasibility of a large randomized controlled trial (RCT) testing the effect of ketamine as an adjunct analgosedative compared with standard of care alone as a control group (CG) in critically ill patients with mechanical ventilation (MV). We also provided preliminary evidence on clinically relevant outcomes to plan a larger trial.
\end{abstract}

Material and methods: Pilot, active-controlled, open-label RCT was conducted at medical, surgical, and transplant ICUs at a large tertiary and quaternary care medical institution (King Faisal Specialist Hospital and Research Center, Saudi Arabia). The study included adult patients who were intubated within $24 \mathrm{~h}$, expected to require MV for the next calendar day, and had institutional pain and sedation protocol initiated. Patients were randomized in a 1:1 ratio to adjunct ketamine infusion $1-2 \mu \mathrm{g} / \mathrm{kg} / \mathrm{min}$ for $48 \mathrm{~h}$ or CG alone.

Results: Of 437 patients screened from September 2019 through November 2020, 83 (18.9\%) patients were included (43 in CG and 40 in ketamine) and 352 (80.5\%) were excluded. Average enrollment rate was 3-4 patients/month. Consent and protocol adherence rates were adequate ( $89.24 \%$ and $76 \%$, respectively). Demographics were balanced between groups. Median MV duration was 7 (interquartile range [IQR] 3-9.25 days) in ketamine and 5 (IQR 2-8 days) in CG. Median VFDs was 19 (IQR 0-24.75 days) in ketamine and 19 (IQR 0-24 days) in the CG $(p=0.70)$. More patients attained goal Richmond Agitation-Sedation Scale at 24 and $48 \mathrm{~h}$ in ketamine (67.5\% and $73.5 \%$, respectively) compared with CG (52.4\% and 66.7\%, respectively). Sedatives and vasopressors cumulative use, and hemodynamic changes were similar. ICU length-of-stay was 12.5 (IQR 6-21.2 days) in ketamine, compared with 12 (IQR 5.5-23 days) in CG. No serious adverse events were observed in either group.

Conclusions: Ketamine as an adjunct analgosedative agent appeared to be feasible and safe with no negative impact on outcomes, including hemodynamics. This pilot RCT identified areas of improvement in study protocol before conducting a large, adequately powered, multicenter RCT which is likely justified to investigate ketamine association with patient-centered outcomes further.

\footnotetext{
*Correspondence: mamer@kfshrc.edu.sa

1 Pharmaceutical Care Division, King Faisal Specialist Hospital

and Research Center, (MBC \# 11), PO Box 3354, Riyadh 11211, Kingdom

of Saudi Arabia

Full list of author information is available at the end of the article
} original author(s) and the source, provide a link to the Creative Commons licence, and indicate if changes were made. The images or other third party material in this article are included in the article's Creative Commons licence, unless indicated otherwise in a credit line to the material. If material is not included in the article's Creative Commons licence and your intended use is not permitted by statutory regulation or exceeds the permitted use, you will need to obtain permission directly from the copyright holder. To view a copy of this licence, visit http://creativecommons.org/licenses/by/4.0/. The Creative Commons Public Domain Dedication waiver (http://creativeco mmons.org/publicdomain/zero/1.0/) applies to the data made available in this article, unless otherwise stated in a credit line to the data. 
Trial registration ClinicalTrials.gov: NCT04075006. Registered on 30 August 2019. Current controlled trials: ISRCTN14730035. Registered on 3 February 2020

Keywords: Ketamine, Critical care, Sedation, Mechanical ventilation, Standard of care, ATTAINMENT, Pilot

\section{Background}

Analgo-sedation or analgesia-first sedation has gained popularity in recent years [1]. This approach has been developed to decrease sedative use, and facilitate mechanical ventilation (MV) weaning [2]. Data on ideal sedatives in intensive care unit (ICU) for mechanically ventilated, and hemodynamically unstable patients are limited. Ketamine has a favorable hemodynamic, analgesic, and adverse effect profile, making it attractive as an analgosedative agent [3, 4]. It inhibits $N$-methyl-D-aspartate (NMDA) receptors and activates opioid $\mu$ - and $\kappa$-receptors [5]. Anesthetists have long used ketamine for acute and chronic pain, procedural sedation, and rapid sequence intubation. It has also been used in postoperative pain control in surgical and trauma patients (as part of multimodal opioid-sparing analgesia in enhanced recovery after surgery), status asthmatics, status epilepticus, alcohol withdrawal, and agitation [6, 7].

Ketamine does not appear to have potential side effects of nonsteroidal anti-inflammatory drugs or opioids negative effects on $\mu$ receptors of gastrointestinal tract associated with ileus [8-10]. Studies to control acute pain in traumatic rib fractures of severely injured individuals at sub-anesthetic doses resulted in reduction of pain scale score and morphine-equivalent dose [11, 12]. Its use has been extended during coronavirus disease (COVID-19) pandemic due to a shortage of other sedatives to keep patients on MV comfortable and synchronous [13, 14]. Ketamine has not been associated with chest wall rigidity precipitating insufficient ventilation, which has occasionally been described with fentanyl [15]. Additionally, propofol and dexmedetomidine-associated hypotension may necessitate vasopressor support which may exclude patients from qualifying for COVID-19 antiviral medication (remdesivir), making ketamine an attractive alternative [16].

There is an increasing body of literature on ketamine use at ICU to reduce sedative requirements and maintain patients within target Sedation-Agitation Scale goal [17-20]. However, evidence provided in Pain, Agitation-Sedation, Delirium, Immobility, and Sleep Disruption (PADIS) guideline supporting its use in mechanically ventilated patients was insufficient due to limited number of randomized controlled trials (RCTs) [1,21]. A trial by Guillou et al. showed a reduction in opioid consumption with low-dose ketamine infusion for $48 \mathrm{~h}$ [22]. However, patients in this trial underwent postoperative abdominal surgery and were able to use patient-controlled analgesia. It is difficult to extrapolate these findings to mechanically ventilated patients who are unable to self-report pain and have a higher severity of illness. Data on whether ketamine affects patient-centered outcomes and its safety in RCTs for critically ill patients with MV, as compared with standard of care, are unclear and have been identified as a research priority. Accordingly, we evaluated the feasibility of an analgosedative adjunct ketamine infusion in mechanically ventilated ICU patients (ATTAINMENT trial) compared to standard of care alone as a control group (CG) using an external, pilot clinical trial design. We also provided preliminary evidence on clinically relevant outcomes for the larger upcoming main trial. We hypothesized that if the study is feasible, then the main, future trial could be conducted to examine ketamine effect on patient-centered outcomes such as duration of MV with an acceptable safety profile compared to CG.

\section{Materials and methods}

This was an investigator-initiated, pilot, single-center, parallel-group, open-label RCT (Registered with ClinicalTrials.gov: NCT04075006, current controlled trials: ISRCTN14730035, and Saudi Food and Drug Authority 19063002). The study was approved by King Faisal Specialist Hospital and Research Center (KFSH\&RC) Institutional Review Board (IRB) (Riyadh, SA, IRB\# 2191187) and full study protocol was published previously [23]. The trial was conducted according to CONSORT guidelines for pilot and feasibility trials and reported according to pilot study checklist [24]. Participants were recruited from KFSH\&RC, a major referral center that provides tertiary and quaternary care.

Patients were eligible if they were admitted to any of three adult ICUs (medical, surgical, and transplant ICU), intubated within previous $24 \mathrm{~h}$ and expected to continue on MV next calendar day, initiated on institutional pain and sedation protocol, and no objection from ICU attending or primary treating team. Recruitment began in September 2019 and was completed in November 2020. Patients were excluded if they had history of dementia or psychiatric disorders, or were comatose on admission due to hepatic encephalopathy. Full inclusion and exclusion criteria are detailed in Additional file 1: Table S1. Our research coordinators, along with local principal investigators screened patients for eligibility by using an electronic screening form in Research 
Electronic Data Capture (REDCap). Once eligibility criteria were met, informed consent was obtained. Given the need to enroll patients in expedited manner within $24 \mathrm{~h}$ window, verbal consent from surrogate decision-maker (SDM) was allowed and documented in electronic medical records (EMR). Written consent was obtained as soon as SDM became available.

\section{Randomization procedure and treatment allocation}

Patients were randomized in 1:1 allocation using a computer-generated, pre-determined randomization list created by an independent biostatistician; no stratification was performed. Group allocation was concealed until after randomization. Investigators were masked to outcomes data during the trial. Although this was an open-label, patients and families were unaware of group assignment. Additional file 1: Figure S1A, B summarizes treatment algorithm. After randomization, CG was started on KFSH\&RC ICU analgesia and sedation protocol. Since it was a nurse-driven protocol, treating team placed an order regarding target Richmond Agitation-Sedation Scale (RASS), and sedatives infusions were adjusted according to RASS target by bedside ICU nurse. For those randomized to intervention group, ketamine $1-2 \mu \mathrm{g} / \mathrm{kg} / \mathrm{min}$ was added as an adjunct for $48 \mathrm{~h}$ and could be weaned off earlier in preparation for extubation. Since this was a pilot, feasibility trial, there was no further intervention after $48 \mathrm{~h}$; however, clinical outcomes and adverse events (AEs) were monitored up to day 28. Ketamine dose was reported in $\mu$ g per kilogram of actual body weight per min as per institutional practice. Other aspects of care, including fluid management, vasopressors use, blood products, enteral nutrition, and early mobilization at discretion of treating team, were similar in both groups. Septic patients were managed according to latest survival sepsis campaign guidelines. Patient-ventilator asynchrony was systematically assessed and managed through inter-professional collaboration by prioritizing analgesia, and management of $M V$ to avoid unnecessary use of neuromuscular blockers (NMB). Spontaneous awakening trial (SAT) was assessed every morning with SAT safety screen unless patients were receiving sedative infusion for status epilepticus or started on NMB post-randomization. Patients who passed SAT were immediately managed using spontaneous breathing trial protocol. Both groups received basic analgesic regimen that included paracetamol and epidural analgesia for hyperthermic intraperitoneal chemotherapy (HIPEC) patients. If delirium treatment was needed, non-pharmacological measures (reassurance or mobilization, and family support) were applied first. If this was insufficient, the protocol allowed antipsychotics use and decision was left to ICU physician.

\section{Outcome measures and data collection}

Primary aim of this pilot trial was feasibility assessed by evaluating consent rate, recruitment success, and protocol adherence. Consent rate was deemed to be adequate if $>70 \%$ of SDMs or patients chose to participate upon being approached. Successful recruitment was defined as $>3$ patients enrolled per month. Protocol adherence was defined as $>75 \%$ of protocolized intervention and assessment of protocol deviation and violation [23]. We conducted educational sessions for clinicians, nurses, and hospital pharmacies to facilitate implementation of protocol. Protocol deviation was also defined as not starting ketamine immediately after randomization (ideally within $4 \mathrm{~h}$ ) due to pharmacy delay or non-placement of ketamine order. Feasibility thresholds (progression criteria) were pre-specified as a priori by investigators and study team after discussion with IRB. Those progression criteria were another critical decision in pilot sample size calculation. Thresholds were chosen after examining other pilot feasibility studies of complex interventions (defined as interventions with several interacting components) and summarized in Additional file 1: Table S2 [25-27].

Clinically relevant primary and co-primary outcomes for the main upcoming trial were median duration of $\mathrm{MV}$, and ventilator-free days (VFDs) up to day 28. This outcome was chosen as patient-centered outcome and influenced by mortality [28]. Other clinical outcomes included the following up to 28 days: ICU and hospital length-of-stay (LOS), mortality rate, and percentage of AEs. We recorded baseline demographics, comorbidities, reasons for ICU admission, and assessed severity of illness with Acute Physiology and Chronic Health Evaluation (APACHE) II and Sequential Organ Failure Assessment (SOFA). We collected proportion and cumulative use of vasopressors, sedatives and analgesics [fentanyl, propofol, midazolam, and dexmedetomidine], and antipsychotics over $48 \mathrm{~h}$ postrandomization. Data on sedatives administered outside ICU during anesthesia or intraoperative were not collected. Presence of delirium was assessed using confusion assessment method for ICU (CAM-ICU), which was measured at baseline and $48 \mathrm{~h}$ post-randomization. If CAM-ICU scores were not available, an electronic progress note was reviewed to detect any evidence of delirium. Hemodynamic parameters [heart rate (HR) and mean arterial pressure (MAP)] were collected $48 \mathrm{~h}$ post-randomization. Hemodynamic changes were defined as presence of tachycardia, hypertension, and hypotension. Details about variables collected and their definitions are available in Additional file 1: Table S3. Data were stored online in REDCap and data quality assessments were executed routinely. 


\section{Statistical analysis}

Following the recommended rules for pilot trial sample size calculation when standardized effect size is unknown but expected to be small, 40 participants per group was recommended [29]. This sample size is considered to be sufficient assuming a protocol adherence of at least $75 \%$ to estimate proportion within 10\% of true rate with $95 \%$ confidence. Details on statistical plan were published previously [23]. Statistician was blinded to group allocation and performed statistical analyses using $\mathrm{R}$ statistical software Version 3.5.0 (R Foundation, Vienna, Austria). Exploratory clinical outcomes analysis included all patients who were enrolled, randomly assigned, and received at least one dose of study medication, constituting modified intention-to-treat (mITT) population. Categorical variables were summarized as counts and percentages. Continuous variables were summarized using either mean $\pm \mathrm{SD}$ or median and interquartile range (IQR), according to normality testing (using Shapiro-Wilk test and histograms). Chi-square test was used to compare categorical variables. Unpaired $t$-test or Mann-Whitney test was used to compare continuous variables. Sensitivity analysis for sedative and vasopressor requirements, excluding patients started on NMB post-randomization, was conducted. We also performed an additional post hoc sensitivity analysis in per-protocol population, defined as mITT population after exclusion of subjects who did not complete $48 \mathrm{~h}$ post-randomization [27]. We ensured immediate data entry and identified missing data quickly, and issues were resolved promptly. Thus, no imputation for missing variables was done. We set statistical significance to two-sided $p$ value of 0.05 .

\section{Results}

From September 2019 through November 2020, a total of 437 patients were screened; 83 (18.9\%) patients met inclusion criteria and 352 (80.5\%) were excluded. Among screened patients, 88 (20.1\%) did not meet eligibility criteria, mainly because they were expected to require MV for $<24 \mathrm{~h}$. Among included patients, 43 were in CG and 40 were included in ketamine group in mITT analysis. Participants' flow through the trial is shown in Fig. 1.

Baseline demographics are described in Table 1 and Additional file 1: Table S4. Median age was 60 years, with a higher proportion of males and medical ICU patients. Overall, demographic characteristics were balanced between groups, except for prevalence of chronic obstructive pulmonary disease, which was higher in CG. We included a wide variety of ICU admission diagnoses and among those randomized to ketamine, $55 \%$ had acute respiratory distress syndrome (ARDS) and about 25\% were recipients of solid organ transplants or had solid malignancy. Other primary reasons for ICU admission included HIPEC (3 patients: 2 in ketamine and 1 in CG), COVID-19 pneumonia (2 patients: one in each group), and sickle cell disease (1 patient in ketamine). Ketaminetreated patients were noted to have higher median lactate level (2.2 [IQR $1.58-3.4 \mathrm{mmol} / \mathrm{L}] p=0.004)$. Median number of hours of ICU admission before study enrollment was $13 \mathrm{~h}$ (IQR 6-21.15) in CG and $15 \mathrm{~h}$ (IQR $12-21)$ in ketamine $(p=0.17)$. Post-randomization, NMB was initiated in $12.5 \%$ of ketamine-treated patients compared to $4.65 \%$ in CG $(p=0.25)$.

\section{Feasibility outcomes}

Average patients enrollment was 3-4 patients/month. Consent rate was adequate; $89.24 \%$ of SDMs or patients chose to participate when approached for consent. Recruitment rate decreased significantly during COVID19 pandemic and was halted for 1 month. We resumed recruitment at a slower rate in March 2020, with an average of 1-2 patients/month. In total, $12 \%$ of patients were enrolled outside traditional working hours (on weekends or night shifts). This process was facilitated through close collaboration with on-call ICU physician. Two (2.4\%) patients were excluded post-randomization, yielding a retention rate of $97.6 \%$. Protocol adherence was $76 \%$ and median hours from consent or enrollment until ketamine started was $4.25 \mathrm{~h}$ [IQR 2.08-5.88]. Reasons of protocol non-adherence are described in Table 2.

\section{Exploratory clinically relevant outcomes}

Clinical and safety outcomes are summarized in Table 3. Median duration of MV on day 28 was 7 days in ketamine group (IQR 3-9.25) compared to 5 days in CG group (IQR 2-8). Median distribution of VFDs at day 28 was 19 days in both groups $(p=0.70)$. Median duration of ICU LOS was comparable between groups. More patients in ketamine achieved goal RASS at 24 and $48 \mathrm{~h}(67.5 \%$ and $73.5 \%$, respectively) compared to CG $(52.4 \%$ and $66.7 \%$, respectively). Median RASS was -2 at baseline, which gradually increased to -1 post-randomization, indicating light sedation and ability of patients to make eye contact with verbal stimulation. Thirty-six (43.37\%) patients underwent CAM-ICU assessment within $48 \mathrm{~h}$ post-randomization, of which $2(5 \%)$ were positive in ketamine. Proportion of patients who did not complete $48 \mathrm{~h}$ of the trial was higher in ketamine (37.5\%) than in CG (11.63\%) and main reason was weaning off sedation in preparation for extubation. Antipsychotics were started in 3 ketamine-treated patients compared to 4 patients in CG $(p=1)$. Dexmedetomidine initiation within $48 \mathrm{~h}$ post-randomization was similar between groups. Higher frequency of hypersalivation and frequent suctioning was observed in CG arm. Regarding hemodynamic changes 


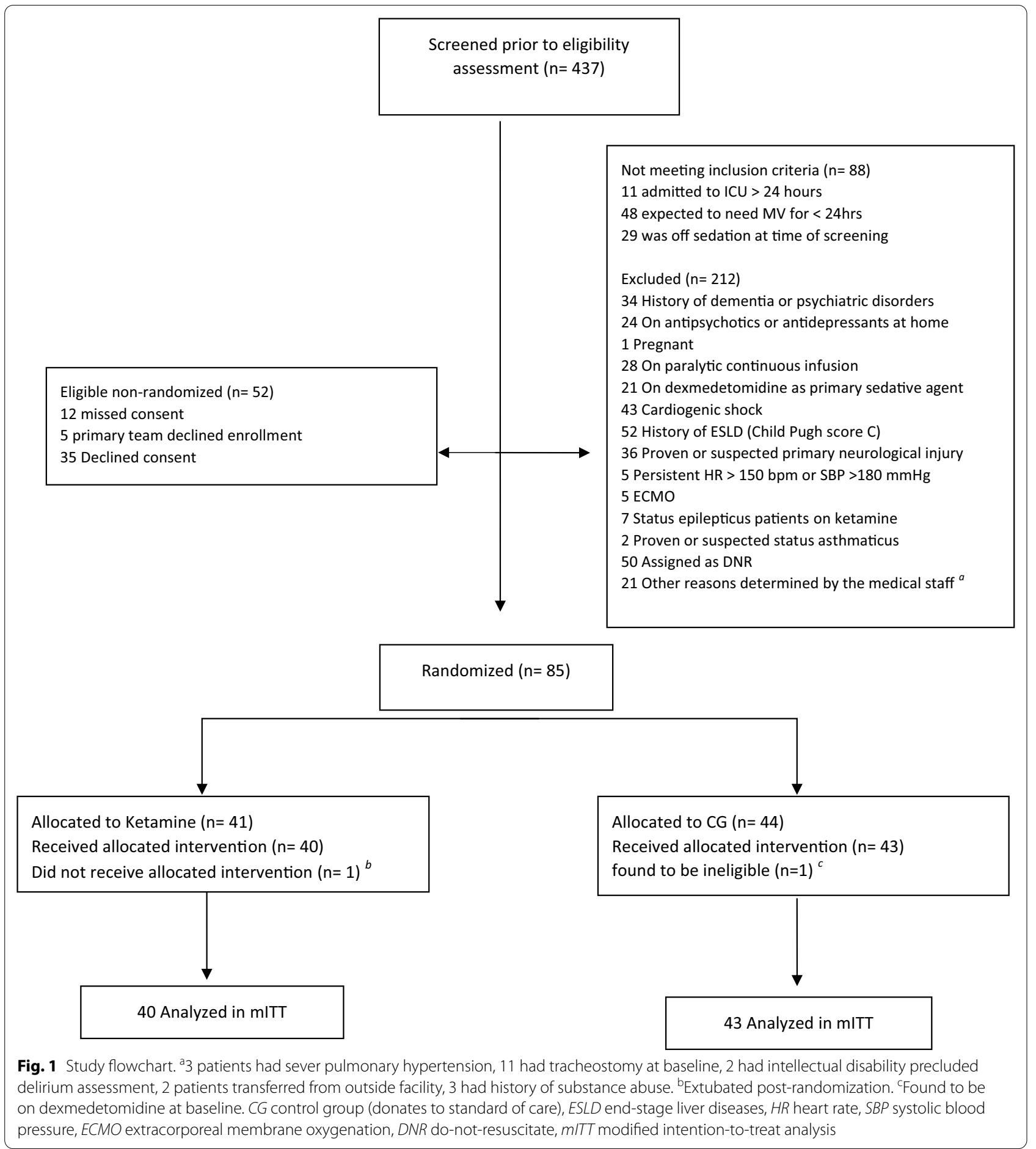

in HR and MAP at 24 and $48 \mathrm{~h}$, we found no difference between groups. The 28-day mortality rate was 11 (27.5\%) in ketamine compared with 14 (32.6\%) in CG, $p=0.79$. Data Safety Monitoring Committee reviewed all deaths, and all were determined to have been due to underlying disease, with participation in trial not being a contributing factor. Additional details on safety outcomes and subgroup analysis are available in Additional file 1: Table S5, Figures S2, S3.

Sedation and vasopressors requirements are summarized in Table 4. There was no difference in baseline values of vasopressor and sedative requirements 
Table 1 Demographic and baseline characteristics

\begin{tabular}{|c|c|c|c|c|}
\hline Variables & All $(N=83)$ & CG $(N=43)$ & Ketamine $(N=40)$ & $p$ \\
\hline Age, years & $61(44.5-71)$ & $61(47.5-70)$ & $59(40.5-73)$ & 0.61 \\
\hline Male, N (\%) & $51(61.4)$ & $25(58.1)$ & $26(65.0)$ & 0.68 \\
\hline Weight, kg & $65(50.7-73.2)$ & $61.8(47.5-69.4)$ & $67.5(51.9-81.2)$ & 0.09 \\
\hline ICU type, $N(\%)$ & & & & 0.74 \\
\hline Medical & $40(48.2)$ & $19(44.2)$ & $21(52.5)$ & \\
\hline Surgical & $22(26.5)$ & $12(27.9)$ & $10(25)$ & \\
\hline Transplant & $21(25.3)$ & $12(27.9)$ & $9(22.5)$ & \\
\hline \multicolumn{5}{|l|}{ The primary reason for ICU admission, $N(\%)$} \\
\hline Sepsis or septic shock & $25(30.1)$ & $14(32.6)$ & $11(27.5)$ & 0.79 \\
\hline Acute respiratory distress syndrome & $50(60.2)$ & $28(65.1)$ & $22(55)$ & 0.47 \\
\hline Cardiovascular & $8(9.64)$ & $3(6.98)$ & $5(12.5)$ & 0.47 \\
\hline Gastrointestinal & $6(7.23)$ & $5(11.6)$ & $1(2.5)$ & 0.20 \\
\hline Neurological & $10(12)$ & $5(11.6)$ & $5(12.5)$ & 1 \\
\hline Trauma & $2(2.41)$ & $1(2.33)$ & $1(2.5)$ & 1 \\
\hline \multicolumn{5}{|l|}{ Comorbidities, N (\%) } \\
\hline COPD & $6(7.23)$ & $6(14)$ & $0(0)$ & 0.03 \\
\hline Mild liver dysfunction [Child-Pugh score A, B] & $9(10.8)$ & $6(14)$ & $3(7.5)$ & 0.49 \\
\hline Diabetes & $28(33.7)$ & $17(39.5)$ & $11(27.5)$ & 0.35 \\
\hline CKD & $16(19.3)$ & $9(20.9)$ & $7(17.5)$ & 0.91 \\
\hline Solid malignancy & $24(28.9)$ & $14(32.6)$ & $10(25)$ & 0.61 \\
\hline Hematological malignancy & $14(16.9)$ & $4(9.3)$ & $10(25)$ & 0.11 \\
\hline Recipient of solid organ transplantation & $21(25.3)$ & $11(25.6)$ & $10(25)$ & 1 \\
\hline HSCT & $7(8.43)$ & $3(6.98)$ & $4(10)$ & 0.71 \\
\hline HIV/AIDS & $1(1.2)$ & $0(0)$ & $1(2.5)$ & 0.48 \\
\hline Hypertension & $24(28.9)$ & $11(25.6)$ & $13(32.5)$ & 0.65 \\
\hline Neuromuscular blockers post-randomization, N (\%) & $7(8.43)$ & $2(4.65)$ & $5(12.5)$ & 0.25 \\
\hline SOFA score & $8(5-10)$ & $8(6-9)$ & $8(5-10)$ & 0.87 \\
\hline APACHE II & $20(13-26)$ & $19(14-25)$ & $20.5(13-26.75)$ & 0.83 \\
\hline Lactate at baseline, $\mathrm{mmol} / \mathrm{L}$ & $1.8(1.2-3.05)$ & $1.4(1-2.3)$ & $2.2(1.58-3.4)$ & 0.004 \\
\hline $\mathrm{PO}_{2} / \mathrm{FiO}_{2}$ ratio & $152(94.1-294)$ & $144(88.9-263)$ & $156(99.2-314)$ & 0.77 \\
\hline PRE-DELIRIC score ${ }^{\mathrm{a}}(\%)$ & $20(12-33)$ & $20(12-36)$ & $20(13-28)$ & 0.68 \\
\hline Heart rate $(H R)$, beats/min & $93(80-106)$ & $91(79-105)$ & $93.5(81.5-106)$ & 0.48 \\
\hline Mean arterial pressure (MAP), mmHg & $77(69-89.5)$ & $77(69.5-88.5)$ & 76.5 (65.5-91) & 0.87 \\
\hline
\end{tabular}

Data presented as $n(\%)$, mean $\pm \mathrm{sd}$, or median (interquartile range)

APACHE II Acute Physiology and Chronic Health Evaluation II, CKD Chronic kidney disease, CG control group (donates to standard of care), COPD chronic obstructive pulmonary disease, $\mathrm{FiO}_{2}$ fraction of inspired oxygen, $\mathrm{HR}$ heart rate, HIV/AIDS human immunodeficiency virus infection and acquired immunodeficiency syndrome, HSCT hematopoietic stem cell transplantation, MAP mean arterial pressure, $\mathrm{PaO}_{2}$ partial pressure of oxygen, PRE-DELIRIC prediction of delirium in ICU patients, SOFA Sequential Organ Failure Assessment

${ }^{a}$ Delirium prediction model designed for adult critical care patients $24 \mathrm{~h}$ after ICU admission and used to predict the factors that may influence delirium risk prior to randomization

pre-randomization, except for amount of vasopressin which was higher in ketamine-treated patients (median 39.6, IQR 30.5-64.2 units, $p=0.053$ ). Cumulative use of fentanyl and other sedatives were similar between two groups at $48 \mathrm{~h}$ post-randomization. Similar trends were observed for cumulative vasopressors use in $\mathrm{mg}$ at $48 \mathrm{~h}$ post-randomization. Sensitivity analysis findings, excluding those who started on NMB post-randomization or those who did not complete $48 \mathrm{~h}$ post-randomization, were consistent with primary analysis (Additional file 1: Tables S6-S8).

\section{Discussion}

This pilot RCT explored the feasibility, and informs the design of a larger, well-designed, phase III RCT to investigate ketamine effect on clinically relevant outcomes in ICU patients with MV. Achieving our threshold of recruitment and consent rate demonstrated that 
Table 2 Reasons for protocol non-adherence

\begin{tabular}{|c|c|c|c|}
\hline Variables & All $(N=83)$ & CG $(N=43)$ & Ketamine $(N=40)$ \\
\hline None & $63(76)$ & $32(74.4)$ & $31(77.5)$ \\
\hline Violated inclusion criteria & $1(1.2)$ & $0(0)$ & $1(2.5)$ \\
\hline Violated exclusion criteria & $1(1.2)$ & $1(2.3)$ & $0(0)$ \\
\hline Missed or discontinued trial protocol, $N(\%)$ & $18(21.7)$ & $10(23.3)$ & $8(20)$ \\
\hline \multicolumn{4}{|l|}{ Reasons $^{\mathrm{a}}$} \\
\hline Excessive sedation and patients not in target RASS, N (\%) & $2(2.4)$ & $0(0)$ & $2(5)$ \\
\hline Death at $48 \mathrm{~h}$ post-randomization, $N(\%)$ & $4(4.8)$ & $2(4.7)$ & $2(5)$ \\
\hline Goal of care changed to comfort care, N (\%) & $2(2.4)$ & $1(2.3)$ & $1(2.5)$ \\
\hline Physician decline patient participation post-randomization, N (\%) & $1(1.2)$ & $0(0)$ & $1(2.5)$ \\
\hline Persistent tachycardia within the first $48 \mathrm{~h}$ [HR $>150$ beats/min], $N(\%)$ & $4(4.8)$ & $1(2.3)$ & $3(7.5)$ \\
\hline $\begin{array}{l}\text { Persistent hypertension within the first } 48 \mathrm{~h} \text { [systolic blood pres- } \\
\text { sure } \geq 180 \mathrm{mmHg} \text {, } N(\%)\end{array}$ & $1(1.2)$ & $0(0)$ & $1(2.5)$ \\
\hline New structural brain disease, $N(\%)$ & $4(4.8)$ & $4(9.3)$ & $0(0)$ \\
\hline Unknown reason, $N(\%)$ & $2(2.4)$ & $2(4.7)$ & $0(0)$ \\
\hline
\end{tabular}

CG control group (donates to standard of care), HR heart rate, RASS Richmond Agitation-Sedation Scale

${ }^{\text {a }}$ Patient could have $>1$ reasons

the trial is feasible and acceptable to clinicians, patients, and families. Adherence rate was acceptable (achieving $>75 \%$ in $76 \%$ of total cohort). Barriers were faced during COVID-19 pandemic due to difficulties in continuing under lockdown conditions, infected research staff, shifting staff to cover COVID-19 ICU, and reorientation in clinical trial research towards COVID-19. We were able to improve adherence rate using strategies such as education sessions for clinical staff and routine clinical reminders, including documentation in EMR. We demonstrated that ketamine appeared to be safe, and had a positive effect on some surrogate clinical outcome as the majority of patients achieved target RASS and pain scores. There was no increase in antipsychotics or dexmedetomidine use post-randomization and no notable hemodynamic changes. Moreover, there was no increase in vasopressor requirements post-randomization despite the fact that ketamine-treated patients were sicker at baseline, as evident by higher lactate level and higher vasopressin dose at baseline. We also did not observe notable severe confusion, nightmares, emergence phenomena, or serious AEs associated with ketamine use, which is consistent with the findings reported by Perbert et al. [30]. Median duration of MV and VFDs in our cohort was consistent with that reported in MENDS2 sedation trial; adjusted median, 23.7 days in dexmedetomidine vs. 24 days in propofol [31]. Notably, the majority of our population were from medical ICU and had moderate ARDS, with median baseline $\mathrm{PaO}_{2} / \mathrm{FiO}_{2}$ ratio of 152 . Overall, 28-day mortality rate in our cohort was $30.1 \%$ which is comparable to mortality rate in patients with severe sepsis and shock and all-cause mortality rate reported in more recent sedation trials, such as SPICE III trial (29\% in dexmedetomidine and usual-care) and MENDS2 trial (38\% in dexmedetomidine and propofol) [31-33].

Furthermore, proportion of patients who did not complete $48 \mathrm{~h}$ of the trials was higher in ketamine than CG and main reason was weaning off sedation for extubation. Such a difference is less likely attributed to variations in illness severity as randomization process ensured well-balanced baseline characteristics between groups in terms of ARDS severity, ICU admission reasons, SOFA, and APACHE II scores. This may be explained by the biological plausibility of ketamine in lowering airway resistance, preserving pharyngeal and laryngeal protective reflexes, and increasing lung compliance without causing respiratory depression in slow infusions [4]. The underlying mechanisms are not fully elucidated yet. One hypothesis is that ketamine has anti-cholinergic effect resulting in bronchodilation, which might be beneficial compared to other sedatives. However, we cannot be entirely sure that our findings are a direct consequence of ketamine rather than an independent improvement of clinical conditions or cumulative effect of concomitant therapies and co-interventions.

Ketamine opioid-sparing effect has been demonstrated in some trials and retrospective studies mainly for ICU patients admitted for postoperative reasons and trauma [20, 22, 34]. In contrast, other trials did not show opioidsparing effect $[30,35,36]$ (Additional file 1: Table S9). In our pilot, there was no difference in amount of sedatives or opioids and underlying reasons are likely multifactorial. Firstly, NMB initiation post-randomization was numerically higher in ketamine but statistically 
Table 3 Clinical outcomes

\begin{tabular}{|c|c|c|c|c|}
\hline Variables & All $(N=83)$ & CG $(N=43)$ & Ketamine $(N=40)$ & $p$ \\
\hline \multicolumn{5}{|l|}{ Clinical outcomes } \\
\hline Liberation from MV within 28 days post-intubation, N (\%) & $52(62.7)$ & $27(62.8)$ & $25(62.5)$ & 1 \\
\hline 28-day duration of MV, days & $5(2-9)$ & $5(2-8)$ & $7(3-9.25)$ & 0.15 \\
\hline Duration of MV at ICU discharge/death, days & $8(3-18.5)$ & $7(3-13.8)$ & $9(3-19)$ & 0.32 \\
\hline Ventilation-free days, days ${ }^{\mathrm{a}}$ & $19(0-24)$ & $19(0-24)$ & $19(0-24.75)$ & 0.70 \\
\hline Patients at goal RASS at $24 \mathrm{~h}, \mathrm{~N}(\%)^{\mathrm{b}}$ & $49(59.8)$ & $22(52.4)$ & $27(67.5)$ & 0.24 \\
\hline Patients at goal RASS at $48 \mathrm{~h}, N(\%)^{c}$ & $51(69.9)$ & $26(66.7)$ & $25(73.5)$ & 0.70 \\
\hline Patients at goal pain score at $24 \mathrm{~h}, \mathrm{~N}(\%)^{\mathrm{d}}$ & $80(96.39)$ & $41(95.35)$ & 39 (97.5) & 1 \\
\hline Patients at goal pain score at $48 \mathrm{~h}, \mathrm{~N}(\%)^{\mathrm{d}}$ & $79(95.2)$ & $41(95.3)$ & $38(95)$ & 1 \\
\hline Discharge from ICU, $N(\%)$ & $76(91.6)$ & $40(93)$ & $36(90)$ & 0.71 \\
\hline ICU length-of-stay, days & $12(6-22.5)$ & $12(5.5-23)$ & $12.5(6-21.2)$ & 0.89 \\
\hline Hospital discharge, N (\%) & 79 (95.2) & $41(95.3)$ & $38(95)$ & 1 \\
\hline Hospital length-of-stay, days & $26(13-39)$ & $27(12.5-47)$ & $26(15.8-38)$ & 0.87 \\
\hline \multicolumn{5}{|l|}{ Safety outcomes } \\
\hline CAM-ICU positive, $N(\%)^{e}$ & $2(2.41)$ & $0(0)$ & $2(5)$ & 0.30 \\
\hline Patients who did not complete 48 h of trial, $N(\%)$ & $20(24.1)$ & $5(11.63)$ & $15(37.5)$ & 0.01 \\
\hline \multicolumn{5}{|l|}{ Hemodynamics } \\
\hline $\mathrm{HR}$ at $24 \mathrm{~h}$ & $92(75.5-107)$ & $95(80-107)$ & $83.5(71.8-105)$ & 0.11 \\
\hline HR at $48 \mathrm{~h}$ & $84(72-100)$ & $89(75-104)$ & $82(71-99)$ & 0.31 \\
\hline MAP at $24 \mathrm{~h}$ & $75(64.5-87)$ & $75(62.5-86.5)$ & $74.5(69.5-91.5)$ & 0.31 \\
\hline MAP at $48 \mathrm{~h}$ & $77(65-90)$ & $76(67.5-87)$ & $77.5(64-92.5)$ & 0.50 \\
\hline Uncontrolled agitation, $N(\%)$ & $10(12.05)$ & $4(9.3)$ & $6(15)$ & 0.51 \\
\hline Combative behavior to the nursing staff, $N(\%)$ & $2(2.41)$ & $1(2.33)$ & $1(2.5)$ & 1 \\
\hline Hyper-salivation and frequent suctioning, $N(\%)$ & $22(26.5)$ & $14(32.6)$ & $8(20)$ & 0.29 \\
\hline Antipsychotics within 48 h post-randomization, N (\%) & $7(8.43)$ & $4(9.3)$ & $3(7.5)$ & 1 \\
\hline Use of physical restraint $48 \mathrm{~h}$ post-randomization, $N(\%)$ & $22(26.5)$ & $10(23.3)$ & $12(30)$ & 0.66 \\
\hline 28-day mortality rate, $N(\%)$ & $25(30.1)$ & $14(32.6)$ & $11(27.5)$ & 0.79 \\
\hline
\end{tabular}

Data presented as $n$ (\%), mean $\pm s d$, or median (interquartile range)

CAM-ICU Confusion Assessment Method for the ICU, CG control group (donates to standard of care), HR heart rate, $M V$ mechanical ventilation, $M A P$ mean arterial pressure, RASS Richmond Agitation and Sedation Scale

${ }^{a}$ VFDs were calculated by subtracting number of ventilation days from 28 after assigning VFD $=0$ for patients who died during 28 days

${ }^{\mathrm{b}}$ The RASS measures levels of consciousness (scores range from -5 [unresponsive] to +4 [combative]). Assessed in 82 patients at $24 \mathrm{~h}$ ( $42 \mathrm{CG}$ and 40 ketamine)

'The RASS was assessed in 73 patients at $48 \mathrm{~h}$ (39 CG and 34 in ketamine)

${ }^{\mathrm{d}}$ Assessment of pain was done by Critical Care Pain Observation Tool for pain (CPOT)

e The CAM-ICU, scores delirium as either present [positive] or not present [negative]. Assessments were done when the patient was maximally awake. If in coma, unable to evaluate

insignificant. Additionally, during COVID-19 pandemic, newly hired non-ICU nurses (to cover manpower shortage) could be unaware of study protocol. Hence, efforts to reduce concomitant sedatives or opioids with ketamine perhaps were conservative. Likewise, we hypothesized that severity of illness, insufficient power, and small ketamine dose in our pilot might provide possible additional explanations.

Our pilot trial had several strengths. Firstly, it included high rates of completed follow-up, and relatively comprehensive assessments of AEs associated with ketamine use and its impact on hemodynamic response. We believe that our results provide incremental value in understanding the effects of ketamine. Adherence to mITT principle, randomization, and blinded outcome assessors limited potential sources of bias. Moreover, our trial included diverse ICU populations and we made every effort to include patients within a narrow randomization window (within $24 \mathrm{~h}$ of intubation) to eliminate potential confounders with other co-interventions. Additionally, information on clinical outcomes was necessary to define clinically meaningful effect of ketamine use which will have an implication on sample size calculations for main upcoming RCT. 
Table 4 Cumulative use of analgesics, sedatives, and vasopressors

\begin{tabular}{|c|c|c|c|c|c|c|c|c|}
\hline & \multicolumn{4}{|l|}{ Baseline } & \multicolumn{4}{|c|}{$48 \mathrm{~h}$ post-randomization } \\
\hline & All $(N=83)$ & $\mathrm{CG}(N=43)$ & $\begin{array}{l}\text { Ketamine } \\
(N=40)\end{array}$ & $p$ & All $(N=83)$ & $\mathrm{CG}(N=43)$ & $\begin{array}{l}\text { Ketamine } \\
(N=40)\end{array}$ & $p$ \\
\hline Fentanyl $(\mu \mathrm{g})$ & $1475(681-2600)$ & $1262(488-2612)$ & $1612(1100-2512)$ & 0.17 & $3938(2100-6400)$ & $3817(2220-6140)$ & 4400 (1588-7700) & 0.67 \\
\hline Fentanyl $(\mu \mathrm{g} / \mathrm{kg})$ & $23.4(9.9-39.7)$ & $21(7.37-37.2)$ & $26.4(15.7-43.2)$ & 0.22 & $66.8(26.6-105)$ & $63.5(32.8-97.1)$ & $69.6(22.7-110)$ & 0.69 \\
\hline Propofol (mg) & 755 (172-1738) & $780(150-1425)$ & $640(215-1850)$ & 0.37 & $1990(530-3862)$ & $2091(492-3316)$ & $1815(778-4272)$ & 0.95 \\
\hline Propofol (mg/kg) & $10.9(3.26-24.7)$ & $12.7(2.13-22.2)$ & $10.6(4.38-25.3)$ & 0.63 & $28.4(9.29-59)$ & $28.4(6.59-58.1)$ & $28(9.62-60.9)$ & 1 \\
\hline Midazolam (mg) & $5(3-5.75)$ & $4.75(2-5.38)$ & $5(3-6)$ & 0.54 & $12.5(5.25-101)$ & $7(4.5-76.8)$ & $62.8(17.1-125)$ & 0.11 \\
\hline $\begin{array}{l}\text { Midazolam (mg/ } \\
\mathrm{kg} \text { ) }\end{array}$ & $0.08(0.04-0.13)$ & $0.08(0.04-0.13)$ & $0.08(0.05-0.12)$ & 0.89 & $0.24(0.1-1.25)$ & $0.15(0.08-0.49)$ & $0.85(0.26-1.74)$ & 0.16 \\
\hline $\begin{array}{l}\text { Dexmedetomidine } \\
(\mu \mathrm{g})\end{array}$ & & & & & $667(357-1222)$ & $667(357-1222)$ & 711 (310-1730) & 0.90 \\
\hline $\begin{array}{l}\text { Dexmedetomidine } \\
(\mu \mathrm{g} / \mathrm{kg})\end{array}$ & & & & & $9.34(5.33-22.8)$ & $9.34(5.33-22)$ & $18(4.67-35.5)$ & 0.63 \\
\hline $\begin{array}{l}\text { Norepinephrine } \\
\text { (mg) }\end{array}$ & $5.92(2.5-12.1)$ & $5.92(1.82-10.7)$ & $6.35(3.53-14.2)$ & 0.38 & $9(4.92-28)$ & $8.63(6.13-26)$ & $9.37(4.4-28.4)$ & 0.89 \\
\hline Epinephrine (mg) & $1.17(0.43-1.37)$ & $1.24(0.53-1.5)$ & $0.81(0.47-1.15)$ & 0.36 & $6.09(2-13.1)$ & $29.2(29.2-29.2)$ & $4.04(1.88-11.35)$ & 0.16 \\
\hline $\begin{array}{l}\text { Phenylephrine } \\
\text { (mg) }\end{array}$ & $0.45(0.3-1.1)$ & $0.45(0.3-1.3)$ & $0.45(0.3-0.7)$ & 0.78 & $0.60(0.21-46.8)$ & $36(0.5-57.6)$ & $0.45(0.17-43.9)$ & 0.52 \\
\hline Dopamine (mg) & $133(70.6-203)$ & $133(110-156)$ & $149(86.2-213)$ & 1 & $563(490-676)$ & $563(482-619)$ & $602(546-659)$ & 0.56 \\
\hline Vasopressin (units) & $18.2(11.4-27.9)$ & $12(9.6-15)$ & $39.6(30.5-64.2)$ & 0.05 & 70.8 (30-91.6) & $24(21.6-82.8)$ & $81.6(60-89.6)$ & 0.20 \\
\hline
\end{tabular}

Data presented as median (interquartile range)

Cumulative use at baseline donates to total amount of analgesics, sedatives, and vasopressors from ICU admission till the time of randomization. Cumulative use at $48 \mathrm{~h}$ donates to total amount of analgesics, sedatives, and vasopressors from time of randomization to $48 \mathrm{~h}$ thereafter

CG control group (donates to standard of care)

There are several limitations worth mentioning. This pilot trial had small sample size and was underpowered to detect true differences in clinical outcomes or subgroup analysis. This will be explored further in adequately sized definitive trial. Although our adherence rate met the feasibility threshold, it is possible to achieve high adherence rate than ATTAINMENT study achieved in this clinical population. We will consider additional strategies to increase protocol adherence for definitive trial (printed checklist of study protocol for bedside nurses, and study signs posted in patients' rooms). Furthermore, medications administration in our pilot trial was unblinded which may have influenced overall RASS and CAM-ICU score assessment and physicians behavior. Therefore, we cannot exclude a possible bias related to open-label design. To minimize this bias, outcome adjudicators and patients and their families were blinded to treatment assignments, and study investigators remained blinded to the results until study conclusion.

This pilot trial highlighted areas of improvement in study protocol before launching a large, adequately powered, multicenter RCT which is likely justified to define ketamine role in ICU patients powered to examine patient-centered outcomes. We believe that the trial protocol could be improved by modifying the current ketamine dosing regimen. We chose ketamine dosing at $1-2 \mu \mathrm{g} / \mathrm{kg} / \mathrm{min}$ because majority of ICU population included in our pilot were older (median age 61 years), with renal and hepatic dysfunction, which potentially alters metabolism and excretion of ketamine and its active metabolite, resulting in increased sensitivity to ketamine, prolonged duration, drug accumulation, and possible longer recovery $[37,38]$. Moreover, the dose described here was in agreement with existing literature describing light sedation strategy and 2018 PADIS guideline recommendations $[1,13,18]$. Published data for ketamine doses showed that it can be safely titrated up to $15 \mu \mathrm{g} / \mathrm{kg} / \mathrm{min}$, as needed, to achieve desired level of analgosedation [4, 6]. Additional file 1: Figure S4 describes the proposed treatment algorithm for the definitive trial with a modified ketamine dosing regimen (titrated to effect).

Moreover, we excluded $17 \%$ of patients due to proven or suspected primary neurological injuries such as those with severe traumatic brain injury (TBI) and hydrocephalus. More recent systematic reviews of mixed acute brain populations (subarachnoid hemorrhage, tumors, and TBI) concluded that ketamine had no detrimental effect on intracranial pressure, ICU LOS, or mortality [39]. Therefore, future RCT may also consider modifying eligibility criteria to include neurocritical care patients to maximize generalizability and 
improve enrollment. However, this may have an implication on sample size calculation and power.

Additionally, small observational reports in burn and COVID-19 patients (5 patients) have linked ketamine chronic high doses (16-50 $\mu \mathrm{g} / \mathrm{kg} / \mathrm{min}$ for up to 26 days) with possible cholangiopathy. We have not observed this concern in our cohort and reasons for cholangiopathy in the aforementioned cases were multifactorial (hemodynamic instability, high positive end-expiratory pressures, reducing hepato-splanchnic blood flow, and direct viral cytopathic effect). We will consider vigilant monitoring of liver tests along with other relevant differentials for future RCT protocol to explore this further $[40,41]$.

We also did not collect data on frequency and duration of prone positioning for ARDS patients who were made prone, or median change in $\mathrm{PaO}_{2} / \mathrm{FiO}_{2}$ ratio postrandomization, limiting the ability to determine the real benefit of ketamine in oxygenation post-randomization. Although we made efforts to validate delirium diagnosis and its assessment with CAM-ICU, we had a large proportion of patients (56.6\%) with un-assessed CAMICU. As such, we plan to include data for MV settings after randomization, CAM-ICU assessments, and other co-interventions (e.g., corticosteroids, prone positioning, and diuretics) in main future RCT study protocol. Finally, ketamine duration was limited to $48 \mathrm{~h}$ due to the nature of this pilot trial. This duration was chosen based on available evidence on the use of sedation in critically ill patients with MV [18, 31, 33]. We assumed that majority of patients remain on sedation and MV for $48 \mathrm{~h}$. This assumption aligned with our own clinical experience at bedside, during which we have observed most of patients usually do not require sedation after $48 \mathrm{~h}$ and likely get extubated within this period. Considering ketamine pharmacokinetics (metabolized in liver, generating active compounds norketamine and hydroxynorketamine, and eliminated in urine with an elimination half-life of roughly $1.5-3 \mathrm{~h}$ ), it is likely that carryover effects occurred despite study design for $48 \mathrm{~h}[37,38]$. Nevertheless, longer duration with close monitoring will be investigated in future definitive trial. Moving forward, a multicenter RCT in collaboration with Saudi Critical Care Trials group will be conducted. Data safety monitoring committee and steering committee will oversee the running of the trial and ensure overall safety of participants. An interim analysis with a pre-specified frequency will be carried out for purposes of determining futility or success of treatment and whether early stopping is appropriate. Specification of interim analysis stopping rule and sample size calculation will be reported separately in main RCT study protocol.

\section{Conclusions}

Ketamine is a potentially attractive option for analgosedation. In our pilot trial, ketamine appeared to be safe, and feasible. However, the lessons learned from this pilot will usefully inform the design and study protocol before conducting main, adequately powered, multicenter RCT to shed light on remaining questions and investigate the association with patient-centered outcomes further. Modifying ketamine dosing regimen, some eligibility criteria, and inclusion of additional data (liver function tests, co-interventions and CAM-ICU) were identified as main goals for modifications to improve recruitment and generalizability.

\section{Supplementary Information}

The online version contains supplementary material available at https://doi. org/10.1186/s40560-021-00569-1.

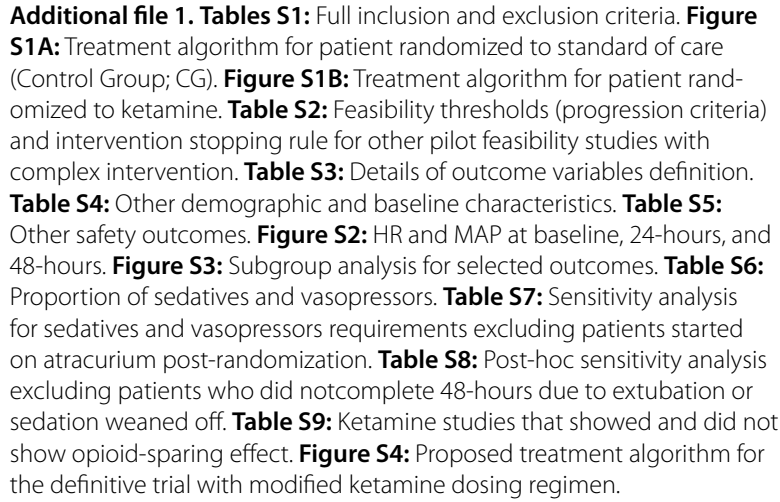

Additional file 1. Tables S1: Full inclusion and exclusion criteria. Figure S1A:Treatment algorithm for patient randomized to standard of care (Control Group; CG). Figure S1B: Treatment algorithm for patient randomized to ketamine. Table S2: Feasibility thresholds (progression criteria) and intervention stopping rule for other pilot feasibility studies with complex intervention. Table S3: Details of outcome variables definition. Table S4: Other demographic and baseline characteristics. Table S5: Other safety outcomes. Figure S2: HR and MAP at baseline, 24-hours, and 48-hours. Figure S3: Subgroup analysis for selected outcomes. Table S6: Proportion of sedatives and vasopressors. Table S7: Sensitivity analysis for sedatives and vasopressors requirements excluding patients started on atracurium post-randomization. Table S8: Post-hoc sensitivity analysis excluding patients who did notcomplete 48-hours due to extubation or sedation weaned off. Table S9: Ketamine studies that showed and did not show opioid-sparing effect. Figure S4: Proposed treatment algorithm for the definitive trial with modified ketamine dosing regimen.

\section{Acknowledgements}

We thank the Saudi Critical Care Trials Group for providing feedback on study proposal, and participants and their families. Without their collective generosity, this trial would not be possible.

\section{Authors' contributions}

MA: led manuscript writing and revisions, conception and design, analytical plan, critical revision of manuscript for important intellectual content, and taking responsibility of the work integrity. KM, and MB: participated in editing manuscript, conception and design, and revision of manuscript for important intellectual content. ED: sample size calculation, analytical plan, and interpretation of data. All authors participated in data acquisition, reviewed the manuscript, approved the final version to be published, and agreed to be accountable for all aspects of the work. All authors read and approved the final manuscript.

\section{Availability of data and materials}

The datasets used and analyzed during the current study are available from the corresponding author on reasonable request.

\section{Declarations}

Ethics approval and consent to participate

The study was approved by King Faisal Specialist Hospital and Research Center (KFSH\&RC) Institutional Review Board (IRB) (Riyadh, SA, IRB\# 2191187) and full study protocol was published previously [23]. Once eligibility criteria were met, informed consent was obtained. Given the need to enroll patients in expedited manner within $24 \mathrm{~h}$ window, verbal consent from surrogate 
decision-maker (SDM) was allowed and documented in electronic medical records (EMR). Written consent was obtained as soon as SDM became available.

\section{Competing interests and source of funding}

The authors declare that they have no competing interests or source of funding.

\begin{abstract}
Author details
'Pharmaceutical Care Division, King Faisal Specialist Hospital and Research Center, (MBC \# 11), PO Box 3354, Riyadh 11211, Kingdom of Saudi Arabia. ${ }^{2}$ College of Medicine, Alfaisal University, Riyadh, Saudi Arabia. ${ }^{3}$ Department of Critical Care Medicine, King Faisal Specialist Hospital and Research Center, Riyadh, Saudi Arabia. ${ }^{4}$ Biostatistics, Epidemiology \& Scientific Computing Department, King Faisal Specialist Hospital and Research Center, Riyadh, Saudi Arabia. ${ }^{5}$ Department of Neurosciences, Residency Training Program, King Faisal Specialist Hospital and Research Center, Riyadh, Saudi Arabia. ${ }^{6}$ Departments of Nursing, King Faisal Specialist Hospital and Research Center, Riyadh, Saudi Arabia.
\end{abstract}

Received: 22 June 2021 Accepted: 25 August 2021

Published online: 30 August 2021

\section{References}

1. Devlin JW, Skrobik Y, Gélinas C, Needham DM, Slooter AJC, Pandharipande PP, et al. Clinical practice guidelines for the prevention and management of pain, agitation/sedation, delirium, immobility, and sleep disruption in adult patients in the ICU. Crit Care Med. 2018;46:e825-73.

2. Shah FA, Girard TD, Yende S. Limiting sedation for patients with acute respiratory distress syndrome - time to wake up. Curr Opin Crit Care. 2017;23:45-51.

3. Whitman CB, Rhodes $\mathrm{H}$, Tellor BR, Hampton NB. Continuous infusion of ketamine for adjunctive sedation in medical intensive care unit: a case series. J Anesthesiol Crit Care Med. 2015;2:1-5.

4. Wieruszewski PM, Leung JG, Nelson S. Ketamine use in the intensive care unit. AACN Adv Crit Care. 2018:29:101-6.

5. Hanidziar D, Bittner EA. Sedation of mechanically ventilated COVID19 patients: challenges and special considerations. Anesth Analg. 2020;13:e40-1.

6. Hurth KP, Jaworski A, Thomas KB, Kirsch WB, Rudoni MA, Wohlfarth KM. The reemergence of ketamine for treatment in critically ill adults. Crit Care Med. 2020;48:899-911.

7. Radvansky BM, Shah K, Parikh A, Sifonios AN, Le V, Eloy JD. Role of ketamine in acute postoperative pain management: a narrative review. Biomed Res Int. 2015;2015:749837.

8. Joachimsson PO, Hedstrand U, Eklund A. Low-dose ketamine infusion for analgesia during postoperative ventilator treatment. Acta Anaesthesiol Scand. 1986;30:697-702.

9. Takieddine SC, Droege CA, Ernst N, Droege ME, Webb M, Branson RD, et al. Ketamine versus hydromorphone patient-controlled analgesia for acute pain in trauma patients. J Surg Res. 2018;225:6-14.

10. Zhao $H$, Yang S, Wang H, Zhang H, An Y. Non-opioid analgesics as adjuvants to opioid for pain management in adult patients in the ICU: a systematic review and meta-analysis. J Crit Care. 2019;54:136-44.

11. Kugler NW, Carver TW, Juul J, Peppard WJ, Boyle K, Drescher KM, et al. Ketamine infusion for pain control in elderly patients with multiple rib fractures: results of a randomized controlled trial. J Trauma Acute Care Surg. 2019;87:1181-8.

12. Carver TW, Kugler NW, Juul J, Peppard WJ, Drescher KM, Somberg LB, et al. Ketamine infusion for pain control in adult patients with multiple rib fractures: results of a randomized control trial. J Trauma Acute Care Surg. 2019;86:181-8.

13. Chanques G, Constantin JM, Devlin JW, Ely EW, Fraser GL, Gélinas C, et al. Analgesia and sedation in patients with ARDS. Intensive Care Med. 2020;46:2342-56.

14. Adams CD, Altshuler J, Barlow BL, Dixit D, Droege CA, Effendi MK, et al. Analgesia and sedation strategies in mechanically ventilated adults with COVID-19. Pharmacotherapy. 2020;40:1180-91.
15. Phua CK, Wee A, Lim A, Abisheganaden J, Verma A. Fentanyl-induced chest wall rigidity syndrome in a routine bronchoscopy. Respir Med Case Rep. 2017;20:205-7.

16. Ammar MA, Sacha GL, Welch SC, Bass SN, Kane-Gill SL, Duggal A, et al. Sedation, analgesia, and paralysis in COVID-19 patients in the setting of drug shortages. J Intensive Care Med. 2021;36:157-74.

17. Erstad BL, Patanwala AE. Ketamine for analgosedation in critically ill patients. J Crit Care. 2016;35:145-9.

18. Groetzinger LM, Rivosecchi RM, Bain W, Bahr M, Chin K, McVerry BJ, et al. Ketamine infusion for adjunct sedation in mechanically ventilated patients. Pharmacotherapy. 2018;38:181-8.

19. Garber PM, Droege CA, Carter KE, Harger NJ, Mueller EW. Continuous infusion ketamine for adjunctive analgosedation in mechanically ventilated, critically ill patients. Pharmacotherapy. 2019;39:288-96.

20. Pruskowski KA, Harbourt K, Pajoumand M, Chui SJ, Reynnolds $\mathrm{HN}$. Impact of ketamine use on adjunctive analgesic and sedative medications in critically ill trauma patients. Pharmacotherapy. 2017:37:1537-44.

21. Bawazeer M, Amer M, Maghrabi K, Al-Sheikh K, Amin R, Rizwan M, et al. Adjunctive continuous ketamine infusion to conventional sedation in mechanically ventilated patients: it is time for a randomized trial. Saudi Crit Care J. 2020:4:5-8

22. Guillou N, Tanguy M, Seguin P, Branger B, Campion J, Mallédant Y. The effects of small-dose ketamine on morphine consumption in surgical intensive care unit patients after major abdominal surgery. Anesth Analg. 2003;97:843-7.

23. Bawazeer M, Amer M, Maghrabi K, Alshaikh K, Amin R, Rizwan M, et al. Adjunct low-dose ketamine infusion vs standard of care in mechanically ventilated critically ill patients at a Tertiary Saudi Hospital (ATTAINMENT Trial): study protocol for a randomized, prospective, pilot, feasibility trial. Trials. 2020;21:288.

24. Thabane L, Ma J, Chu R, Cheng J, Ismaila A, Rios LP, Robson R, Thabane M, Giangregorio L, Goldsmith CH. A tutorial on pilot studies: the what, why and how. BMC Med Res Methodol. 2010;10:1.

25. Craig P, Dieppe P, Macintyre S, Michie S, Nazareth I, Petticrew M. Developing and evaluating complex interventions: the new medical research council guidance. BMJ. 2008;337:a1655.

26. Rochwerg B, Millen T, Austin P, Zeller M, D'Aragon F, Jaeschke R, et al. Fluids in sepsis and septic shock (FISSH): protocol for a pilot randomised controlled trial. BMJ Open. 2017;7:e017602.

27. Vaara ST, Ostermann M, Bitker L, Schneider A, Poli E, Hoste E, et al. Restrictive fluid management versus usual care in acute kidney injury (REVERSE-AKI): a pilot randomized controlled feasibility trial. Intensive Care Med. 2021:47:665-73.

28. Yehya N, Harhay MO, Curley MAQ, Schoenfeld DA, Reeder RW. Reappraisal of ventilator-free days in critical care research. Am J Respir Crit Care Med. 2019;200:828-36.

29. Lancaster GA, Dodd S, Williamson PR. Design and analysis of pilot studies: recommendations for good practice. J Eval Clin Pract. 2004; 10:307-12.

30. Perbert S, Verdonk F, Godet T, Jabaudon M, Chartier C, Cayot S, et al. Low doses of ketamine reduce delirium but not opiate consumption in mechanically ventilated and sedated ICU patients: a randomised double-blind control trial. Anaesth Crit Care Pain Med. 2018;37:589-95.

31. Hughes CG, Mailloux PT, Devlin JW, Swan JT, Sanders RD, Anzueto A, et al. Dexmedetomidine or propofol for sedation in mechanically ventilated adults with sepsis. N Engl J Med. 2021;384:1424-36.

32. Vallet $H$, Schwarz GL, Flaatten $H$, de Lange DW, Guidet B, Dechartres A. Mortality of older patients admitted to an ICU: a systematic review. Crit Care Med. 2021:49:324-34

33. Shehabi Y, Howe BD, Bellomo R, Arabi YM, Bailey M, Bass FE, et al. Early sedation with dexmedetomidine in critically ill patients (SPICE III). N Engl J Med. 2019;380:2506-17.

34. Buchheit JL, Yeh DD, Eikermann $M$, Lin $\mathrm{H}$. Impact of low-dose ketamine on the usage of continuous opioid infusion for the treatment of pain in adult mechanically ventilated patients in surgical intensive care units. J Inten Care Med. 2019;34:646-51.

35. Alshahrani MS, Asonto LP, El Tahan MM, Al Sulaibikh AH, Al Faraj SZ, Al Mulhim AA, et al. Study protocol for a randomized, blinded, controlled trial of ketamine for acute painful crisis of sickle cell disease. Trials. 2019;20:286. 
36. Alshahrani MS, Alsulaibikh AH, ElTahan MM, AlFaraj SZ, AlMulhim AA, AlMaghraby $\mathrm{NH}$, et al. Ketamine use for acute painful crisis of sickle cell disease: a randomized controlled trial (research forum abstract). Ann Emerg Med. 2019;74:S89-90.

37. Power BM, Forbes AM, van Heerden PV, Ilett KF. Pharmacokinetics of drugs used in critically ill adults. Clin Pharmacokinet. 1998;34:25-56.

38. Zanos P, Moaddel R, Morris PJ, Riggs LM, Highland JN, Georgiou P, et al. Ketamine and ketamine metabolite pharmacology: insights into therapeutic mechanisms. Pharmacol Rev. 2018;70:621-60.

39. Gregers MCT, Mikkelsen S, Lindvig KP, Brøchner AC. Ketamine as an anesthetic for patients with acute brain injury: a systematic review. Neurocrit Care. 2020;33:273-82.
40. Meersseman P, Blondeel J, De Vlieger G, van der Merwe S, Monbaliu D, et al. Secondary sclerosing cholangitis: an emerging complication in critically ill COVID-19 patients. Intensive Care Med. 2021;1-4.

41. Deltenre P, Moreno C, Trépo E. Progressive cholangiopathy in COVID-19 patients: other possible diagnoses than ketamine-induced cholangiopathy should be considered. J Hepatol. 2021. https://doi.org/10.1016/j.jhep. 2021.02.036.

\section{Publisher's Note}

Springer Nature remains neutral with regard to jurisdictional claims in published maps and institutional affiliations.
Ready to submit your research? Choose BMC and benefit from:

- fast, convenient online submission

- thorough peer review by experienced researchers in your field

- rapid publication on acceptance

- support for research data, including large and complex data types

- gold Open Access which fosters wider collaboration and increased citations

- maximum visibility for your research: over $100 \mathrm{M}$ website views per year

At BMC, research is always in progress.

Learn more biomedcentral.com/submissions 